\title{
Selecting Design Objectives for an Integrated Guidance System of a Commercial Launch Vehicle with Application of GPS Technologies
}

\author{
Nickolay Zosimovych*
}

\begin{abstract}
Mechanical and Automobile Department, School of Engineering and Technology, Sharda University, Greater Noida, Uttar Pradesh, 201301, India
\end{abstract}

\begin{abstract}
In this article have been proposed the design objectives for an integrated guidance system of a commercial launch vehicle with application of GPS technologies and has been set a technical problem of the conceptual design of an integrated navigation system for the space launch vehicle qualified to inject small artificial Earth satellites into low and medium circular orbits. The conceptual design of the integrated navigation system based on GPS technology involves determination of its structure, models and algorithms, providing the required accuracy and reliability in injecting payloads with due regard to restrictions on weight and dimensions of the system.
\end{abstract}

Keywords: A gimbaled inertial navigation system (GINS), global positioning system (GPS), an inertial navigation system (INS), mathematical model (MM), navigation, pseudo-range, pseudo-velocity, the launch vehicle.

\section{INTRODUCTION}

A key tendency in the development of affordable modern navigation systems is displayed by the use of integrated GPS/INS navigation systems consisting of a gimbaled inertial navigation system (GINS) and a multichannel GPS receiver [1]. The investigations show [2, 3], that such systems of navigation sensors with their relatively low cost are able to provide the required accuracy of navigation for a wide range of highly maneuverable objects, such as airplanes, helicopters, airborne precision-guided weapons, spacecrafts, launch vehicles and recoverable orbital carriers.

The study of applications of GPS navigation technologies for highly dynamic objects ultimately comes to solving the following problems [4]:

1. Creation of quality standards (optimality criteria) for solving the navigation task depending on the type of an object, its trajectory characteristics and restrictions on the weights, dimensions, costs, and reliability of the navigation system.

2. Selection and justification of the system interconnecting the GPS-receiver and GINS: uncoupled, loosely coupled, tightly coupled (ultra-tightly coupled).

3. Making mathematical models (MM) of an object's motion, including models of external factors beyond control influencing object (disturbances). This requires to make two types of object models: the most detailed and complete one, which will be later

\footnotetext{
*Address correspondence to this author at the Mechanical and Automobile Department, School of Engineering and Technology, Sharda University, Greater Noida, Uttar Pradesh, 201301, India; Tel: +91 956055 8421;

E-mail: n.zosimovych@sharda.ac.in
}

included in the model of the environment when simulating the operation of an integrated system, and a so-called on-board model, which is much simpler and more compact than the former one, and will be used in the future to solve the navigation problem being a part of the on-board software.

4. Making MM for GINS considering the use of gyroscopes and accelerometers (i.e. it is required to make a model for navigation measurements supplied by GINS, taking into account systematic (drift) and random measurement errors).

5. Making a model of the navigation field of GPS, including system architecture, a method of calculating ephemeris of navigation satellites in consideration of possible errors, clock drifts on board the navigation satellites, and taking into account the conditions of geometric visibility of a navigation satellite on different parts of the trajectory of a highly dynamic object.

6. Making a model of a multichannel GPS receiver, including models of code measurements (pseudorange and pseudo-velocity) and, if necessary, phase measurements, including the whole range of chance and indeterminate factors beyond control, existing when such measurements are conducted (such as multipath effect).

7. Choosing an algorithm to process measured data in an integrated system in agreement with the speed-ofresponse requirement (the possibility to process data in real time) and demand accuracy in solving a navigation task. 
8. Creating an object-oriented computer complex for the implementation of the above models and algorithms with the objective to model the process of functioning of the integrated navigation system of a highly dynamic object.

Let's consider the above objectives, regarding the peculiarities of the subject of inquiry, namely a commercial launch vehicle, designed to launch payloads into low Earth orbit (LEO) or geostationary orbit (GSO), in more details.

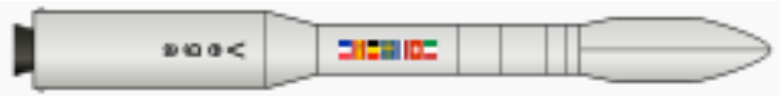

Fig. (1). Launch vehicle Vega (Vettore Europeo di Generazione Avanzata, ASI\&ESA) [5].

Within the framework of this study, we shall consider a light launch vehicle which has been jointly developed by the European Space Agency (ESA) and the Italian Space Agency (ASI) since 1998 (Fig. 1). It is qualified to launch satellites ranging from $300 \mathrm{~kg}$ to $2000 \mathrm{~kg}$ into low circular polar orbits. As a rule, these are low cost projects conducted by research organizations and universities monitoring the Earth in scientific missions as well as spy satellites, scientific and amateur satellites. The main characteristics of the launch vehicle are given in Table $\mathbf{1}$. The launch vehicle Vega [5] is the prototype of the vehicle under development.

The planned payload to be delivered by the launch vehicle to a polar orbit at an altitude of $\sim 700 \mathrm{~km}$ shall be $1500 \mathrm{~kg}$. The launch vehicle is tailored for missions to low Earth and Sun-synchronous orbits. During the first mission, the light class launch vehicle is to launch the main payload, a satellite weighing $400 \mathrm{~kg}$, to an altitude of $1450 \mathrm{~km}$ with an inclination of the orbit $71^{0} 50^{\mathrm{m}}$. Unlike most single-body launchers, this vehicle is to launch several spacecrafts. Here are the main types of spacecrafts that can be a potential payload [1]:

- $\quad$ microsatellites - up to $300 \mathrm{~kg}$;

- $\quad$ mini satellites — between 300 and $1000 \mathrm{~kg}$;

- $\quad$ small satellites — between 1000 and $2000 \mathrm{~kg}$.

The launch vehicle under consideration is the smallest one developed by ESA. We assume that the new launch vehicle will be able to meet the demands of the market for launching small research satellites and will enable universities to conduct research in space. The launcher will be primarily used for satellites that monitor the Earth surface. The injection is conducted according to the most popular and simplest (and the cheapest) scenario [6], more specifically: the instrument unit and the navigation system ride atop the 3rd stage of the launch vehicle.

Thus, launching until separation of the 4th stage carrying payload is conducted in accordance with the data provided by the navigation system which estimates 12 components of the launcher state vector, including position, velocity, orientation angles and angular velocities. Basically, launching may be done upon implementation of any of the possible algorithms, for example, a terminal one, that provides accuracy of the 3rd stage launching to the calculated point of separation of the 4th stage or the traditional algorithm which minimizes the deviation of the center of mass of the launcher from the preselected programmed trajectory [5].

Table 1. Key Specifications of the Vega Launch Vehicle [5]

\begin{tabular}{|c|c|}
\hline Specification & Values \\
\hline \multicolumn{2}{|c|}{ Main technical specifications } \\
\hline Number of stages & 4 \\
\hline Length & $30 \mathrm{~m}$ \\
\hline Diameter & $3 \mathrm{~m}$ \\
\hline \multicolumn{2}{|l|}{ First stage - P80 } \\
\hline Length & $10.5 \mathrm{~m}$ \\
\hline Diameter & $3.0 \mathrm{~m}$ \\
\hline Sustainer Engine & RDTT (solid fuel rocket engine) \\
\hline Thrust & $3040 \mathrm{k} \mathrm{N}$ \\
\hline Burn time & $107 \mathrm{~s}$ \\
\hline Fuel & Solid \\
\hline \multicolumn{2}{|c|}{ Second stage - Zefiro 23} \\
\hline Length & $7.5 \mathrm{~m}$ \\
\hline Diameter & $1.9 \mathrm{~m}$ \\
\hline Sustainer Engine & RDTT (solid fuel rocket engine) \\
\hline Thrust & $1200 \mathrm{kN}$ \\
\hline Burn time & $71.6 \mathrm{~s}$ \\
\hline Fuel & Solid \\
\hline \multicolumn{2}{|c|}{ Third stage - Zefiro 9} \\
\hline Length & $3.85 \mathrm{~m}$ \\
\hline Diameter & $1.9 \mathrm{~m}$ \\
\hline Sustainer Engine & RDTT (solid fuel rocket engine) \\
\hline Thrust & $214 \mathrm{kN}$ \\
\hline Burn time & $117 \mathrm{~s}$ \\
\hline Fuel & Solid \\
\hline \multicolumn{2}{|c|}{ Fourth stage - AVUM } \\
\hline Length & $1.74 \mathrm{~m}$ \\
\hline Diameter & $1.9 \mathrm{~m}$ \\
\hline Sustainer Engine & LRE AVUM \\
\hline Thrust & $2.45 \mathrm{kN}$ \\
\hline Burn time & $315.2 \mathrm{~s}$ \\
\hline Fuel & UDMH \\
\hline Oxidizer & AT \\
\hline
\end{tabular}

The injection sequence which is being described here supposes conducting the following procedures at peak altitude reached by the 3 rd stage, namely the computation of the required orientation of the 4th stage and the computation of the required impulse to transfer the payload carried by the 
4th stage to an orbit of an artificial satellite of the Earth from the final point reached by the 3rd stage. Thus, the transfer of the 4th stage from the end-point of lifting the 3rd stage to an orbit of injecting the payload is performed by the software, i.e. without the use of navigation data, and thus the accuracy of injection of the payload into the required orbit is determined by two factors: the accuracy of lifting the 3rd stage in the predetermined terminal point and the accuracy of the program control in the 4th stage [5].

\section{PROBLEM SETTING}

From the standpoint of the problem concerned, namely the synthesis of the navigational algorithm of the space launcher in the proposed injection sequence we are interested only in the first factor, i.e. accuracy of lifting of the 3rd stage to the point of separation of 4th stage. This accuracy, other conditions being equal, is determined by the precision of solving a navigation task in lifting the 3rd stage in consideration of both components: the center of mass and the velocity of the stage. They predetermine the required impulse for the 4th stage [7].

Thus, we may determine the main criterion of the accuracy of the navigation task in relation to the integrated inertial navigation system of the space launch vehicle: we need to ensure maximum accuracy in determining the position and velocity vectors of the 3rd stage of the launch vehicle in the exo-atmospheric phase of the mission for the selection of navigation coordinate system. Clearly, this accuracy, in its turn, other things being equal, depends upon the accuracy of the initial conditions of travel of the 3rd stage, or in other words, the accuracy of navigation on the previous atmospheric phase of the mission [1].

Consequently, in the case of the proposed injection sequence, the simplest and most obvious criterion for evaluation of the accuracy of the synthesized system should be adopted. It is required to ensure maximum accuracy in determining the vectors of position and the center of mass velocity of the launcher during the flight of the1st-3rd stages, i.e. in atmospheric and exo-atmospheric phases of the mission. This accuracy can be characterized by the value of the dispersions posteriori of the corresponding components of the mentioned vectors [8]. Now let's consider the possible integration schemes for GINS and GPS receiver with respect to this technical problem. As it has been aforementioned, currently we can think of three possible integration schemes as follows [9-13]:

- $\quad$ uncoupled (separated subsystems);

- $\quad$ loosely coupled;

- $\quad$ tightly coupled (ultra-tightly coupled).

Let's consider the peculiarities of these systems.

Uncoupled systems are the simplest option for simultaneous use of INS and GPS receiver (Fig. 2) [14]. Both systems operate independently. But, as INS errors constantly accumulate, it is eventually necessary to make correction of INS according to data provided by the GPS receiver. Creating such architecture requires minimal changes to the hardware and the software.

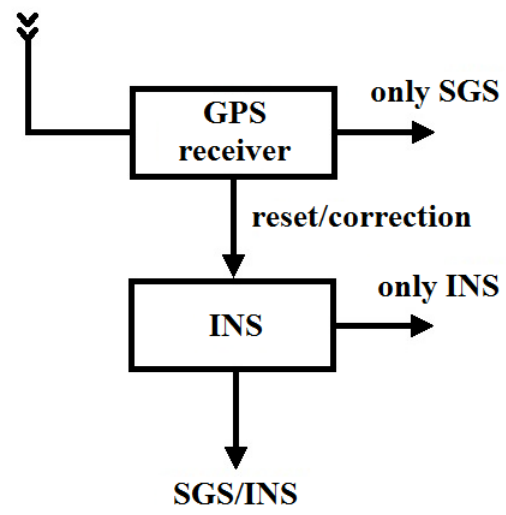

Fig. (2). Uncoupled system with simultaneous use of INS and GPS receiver.

In loosely coupled systems (Fig. 3), GINS and GPS also generate separate solutions, but there is a binding unit in which GPS-based measurements and GINS readouts make assessment of the status vector and make corrections of data provided by GINS [14].

A loosely coupled complex envisages an independent identification of navigation parameters both by GINS and Self-Guided System (SGS). Different navigation parameters (coordinates, velocities) are provided by GINS and SGS. They are then used in the Kalman filter to determine errors occurring in GINS with a purpose of their subsequent compensation.

Such systems usually use two filters: the first one is a part of the satellite receiver and the second one is used for co-operative processing of information. The advantage of this scheme is in high functional reliability of the navigation system. The drawback is in correlation of errors, arriving from SGS to the input of the second Kalman filter and the need of strict synchronization of measurements provided by INS and SGS [14].

In sources loosely coupled systems are divided into three following types [12]: the standard, "aggressive" and the socalled MAGR schemes. The difference between "aggressive" scheme and the standard one is that the former one uses the information on acceleration for extrapolation of navigation sighting executed by SGS provided by GINS in the period between measurements (Fig. 3). The Rockwell MAGR scheme uses inertial measurement from the SGS receiver made in carrier tracking loop (Fig. 4).

In tightly coupled systems (Fig. 4), the role of the INS is reduced only to the measurement of the primary parameters of translational and rotational motions. For this reason, in such systems INS are only inertial measurement units, and the GPS receiver is without Kalman filter. In such a structure, both INS and SGS provide a series of measurements for a common computing unit [14].

Tightly coupled systems are characterized by high accuracy compared with aforementioned systems, and the integrated filter makes it possible to use all available GPS 


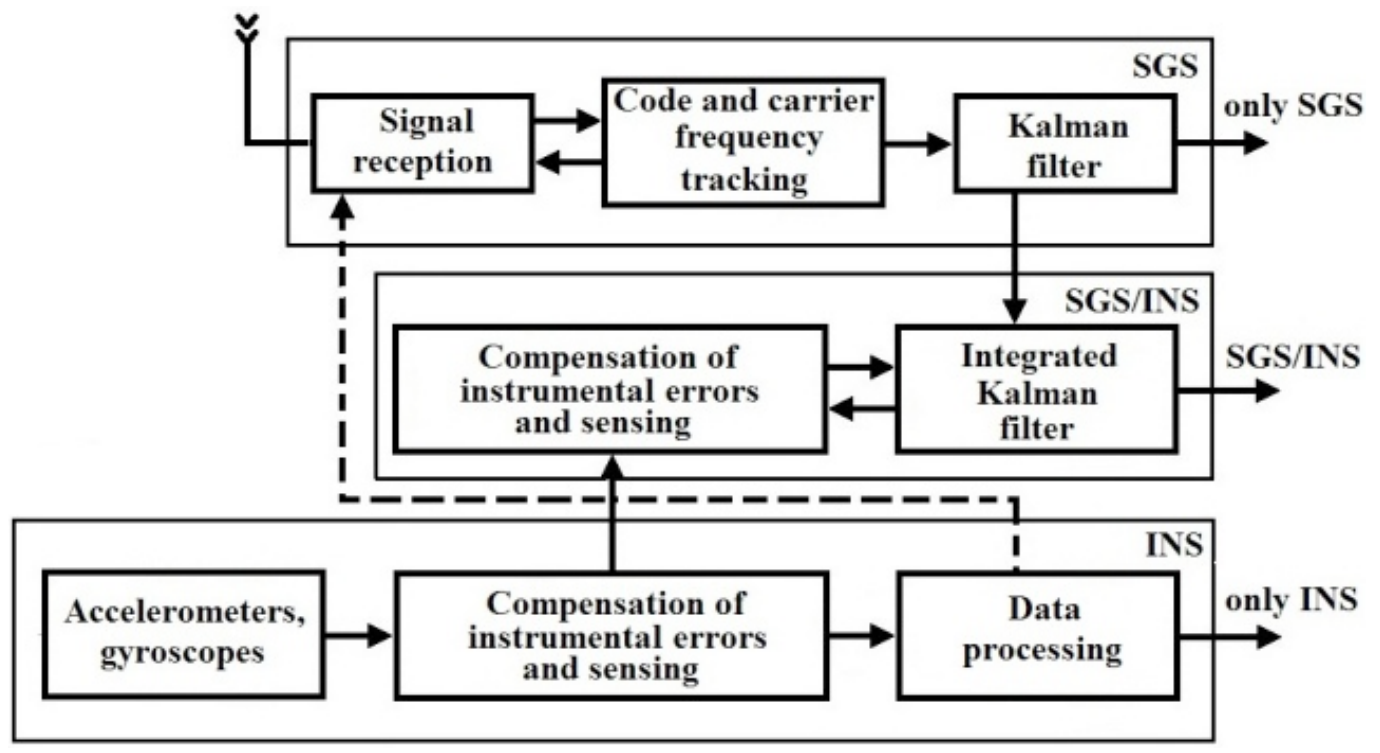

Fig. (3). Loosely coupled system using GINS and GPS.

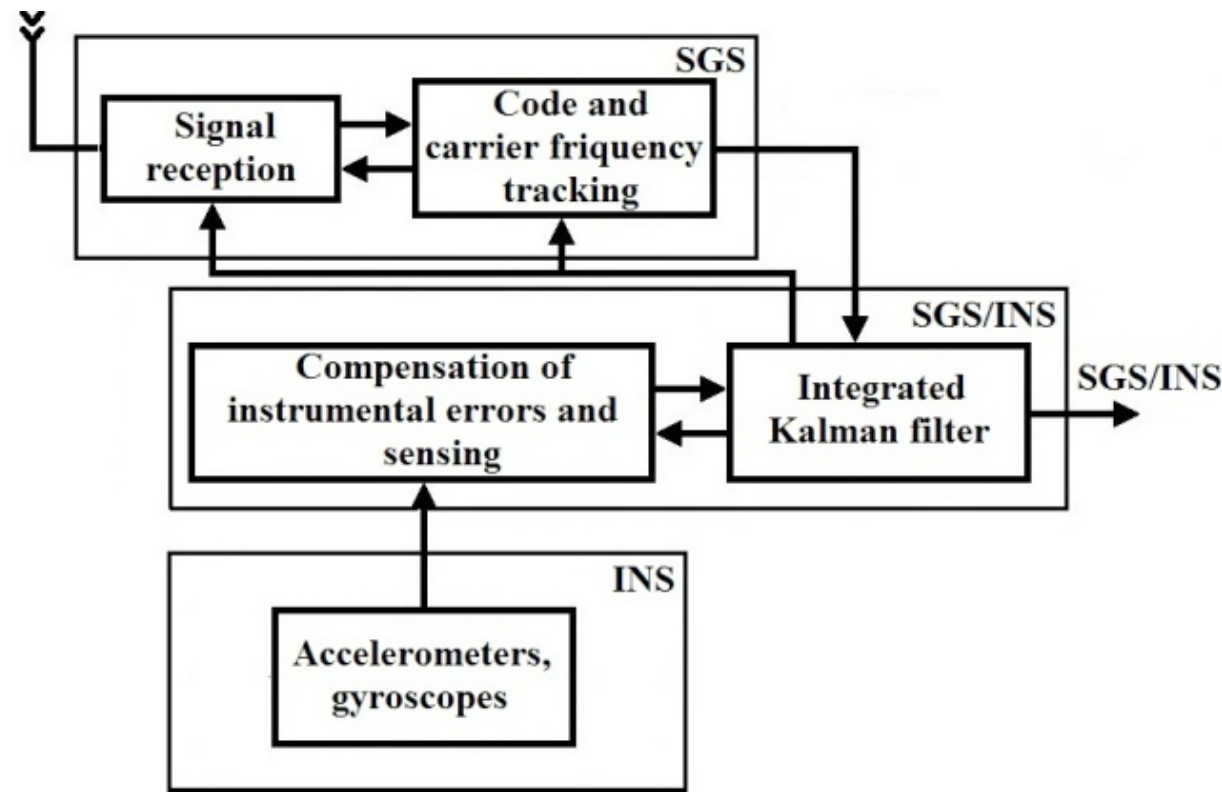

Fig (4). Tightly coupled system using INS and GPS receiver.

satellites in an optimal way, but at cost of the functional redundancy of the system. Tightly coupled systems use the only "evaluator" (as a rule, the Kalman filter) that uses differences between pseudo-ranges and/or pseudo-velocities, calculated (predicted) by INS and measured by Self-Guided System. Advantages of such a scheme are the following:

- $\quad$ the problem of measurement correlation is absent;

- there is no need of synchronization of INS and SelfGuided System as just one clock generator is used;

- $\quad$ search and selection of law quality measurements of pseudo-ranges.

The disadvantages of closely coupled systems are the following:

- the need for special equipment for Self-Guided Systems;
- use of complex equations for measurements;

- low reliability because INS failure may result in failure of the whole system.

The later drawback can be eliminated by introducing a parallel Kalman filter only for Self-Guided System.

Thus, the main differences between a tightly coupled system and a loosely coupled system are as follows:

- $\quad$ use of the INN output information on acceleration in the code and carrier frequency tracking loop. This allows to narrow the loop bandwidth and improve performance and tuning accuracy;

- use of pseudo-ranges and pseudo-velocities (instead of coordinates and velocities) to estimate errors in INS. 


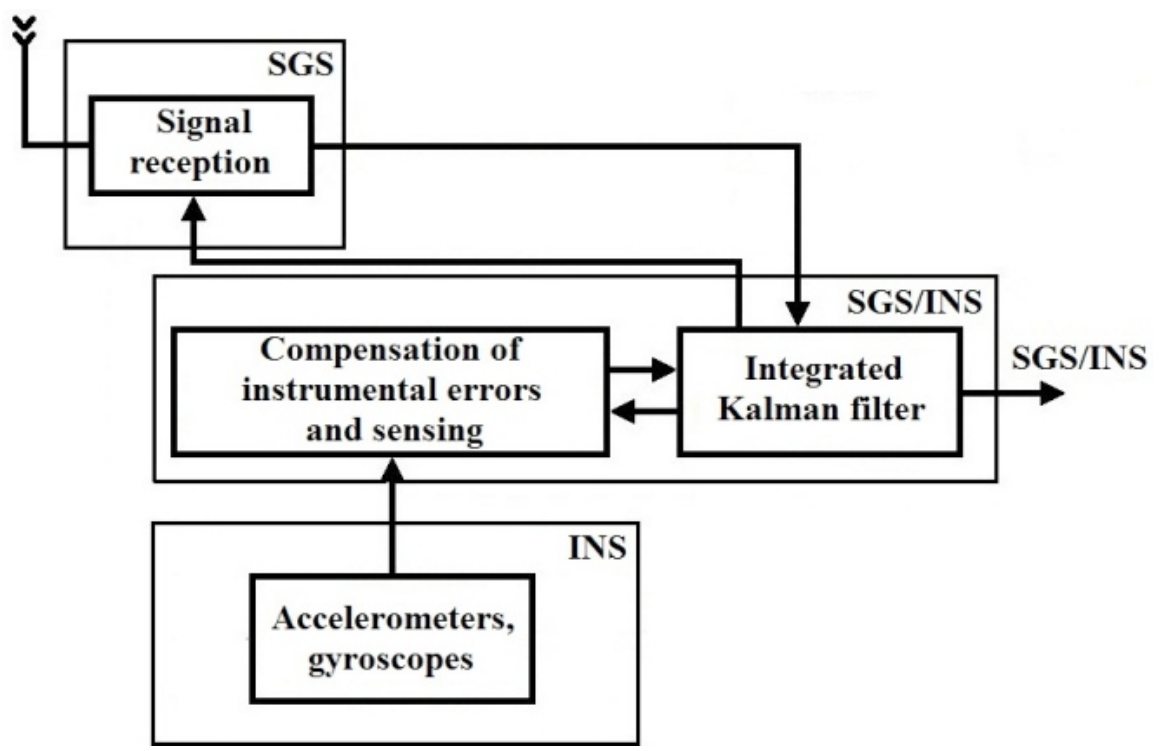

Fig. (5). Ultra-tightly coupled system.

A separate embodiment of the tightly coupled systems is the so-called ultra-tightly coupled systems. In such systems (Fig. 5), estimations are undertaken in the integrated Kalman filter, and the GPS receiver is further simplified [14]. In this case, the Kalman filter is of order 40 and its implementation requires a computer with a very high speed.

The main factors that determine the structure and composition of the navigation system are required accuracy and reliability of navigation parameters within the given limits on the weight, size, power consumption (in some cases - for the time of the system development and operation security) (Table 2). Besides, consideration should also be given to:

- types of objects;

- $\quad$ cost of the complex;

- $\quad$ service conditions;

- $\quad$ possibility of maintenance and repair.

Table 2. The Main Advantages of Integrated Systems

\begin{tabular}{|c|c|}
\hline Factors & Quality Characteristic \\
\hline \hline Accuracy & Substantially \\
\hline Weight & decreasing by $30-70 \%$ \\
\hline Volume & decreasing by $50-60 \%$ \\
\hline Power consumption & decreasing by $25-50 \%$ \\
\hline Reliability & increasing $\approx 2$ times \\
\hline Redundancy level & increasing by $50 \%$ and more \\
\hline Cost & Substantially \\
\hline
\end{tabular}

Proceeding from the above information we may conclude that an integrated navigation system of future launchers should have a structure which, depending on the functionality of SGS receiver, shall allow operating in accordance with the algorithms both as an uncoupled and tightly coupled system. It should be capable of processing coordinates and velocities as well as pseudo-ranges and pseudo-velocities.

The structure of the complex is to be opened to information from other on-board navigation tools and external consumers of navigation information. This may be done by introducing the corresponding input/output ports.

With regard to the above considerations, we propose the following structure of the integrated complex:

- GINS - the main system that provides selfsufficiency and reliability;

- GPS receiver - a device correcting GINS in latitude, longitude, altitude and velocity in three velocity projection components;

- On board computer - carries out a full range of programs providing operation in various modes, in particular, it comprises a Kalman filter algorithm.

Clearly, the first of the above schemes using both GINS and GPS receiver is not acceptable for our task, because here the receiver is not used for calibration (adjustment) of GINS during the mission by evaluating the drift component. As a result, in the absence of GPS-data, errors of GINS grow at the same rate as in the absence of the receiver.

Next, each of the two following schemes of interconnection (uncoupled and tightly coupled) have their advantages and disadvantages regarding the technical problem in question. Indeed, by using a loosely coupled scheme we can implement evaluation of GINS drift components and therefore in the absence of GPS-data, "departure" of GINS will be significantly compensated. Here at the Kalman filter, we shall have comparatively small dimensions in a loosely coupled scheme, i.e. it shall be simple enough for technical implementation. However, with respect to such a highly dynamic object as a launch vehicle 
in the end it turns out that the accuracy of executing a navigation task is determined by errors of a multi-channel receiver. But with regard to peculiarities of object's motions and flight time limitations, this accuracy may not be sufficient to provide the required accuracy of the payload injection because in loosely coupled scheme receiver errors are not evaluated. Which means that the apriori rejection of a tightly coupled scheme as the most challenging to implement is not a sufficient reason? Indeed, if the flight conditions allow us to estimate the actual values of systematic errors in measurement of pseudo-range and pseudo-velocity, the tightly coupled scheme allows us obtain the highest possible accuracy of navigation.

Here, certainly appear additional problems with the big Kalman filter and mathematical models of systematic measurements of pseudo-range and pseudo-velocity caused by atmospheric delays, receiver clock drifts, multipath, etc.

Thus, we conclude that in the present study it is appropriate to examine both schemes of interconnection: tightly and loosely coupled, and based on the results of simulation, conclusions are drawn in favor of one of the possible solutions. Let us briefly examine the scientific and technical problems arising when making the corresponding models and algorithms.

MM of spatial motion of center of mass and relative to center of mass of a solid launch vehicle is well known and widely described in sources. The greatest difficulty in the implementation of such a model as a part of the model of the environment, represents a model of a solid-propellant rocket engine with thrust distribution in respect to the nominal model in mind and the model of stage separation from the point of view of the influence of disturbing moments that arise when dividing into initial conditions of the motion of the next stage.

The key question here is the question of the appropriate level of complexity of the "on-board" model of launcher movement used in the Kalman filter to predict its movement. The answer to this question can also be obtained by simulation of the navigation process.

Mathematical models of GINS are currently also well described in sources, e.g. [15-18]. At the same time MM of GINS drift depends essentially on the type of gyro units and accelerometers used in GINS. In other words, a so-called non-modelable constant is always present in the drift model. It ultimately determines the possibility of GINS alignment during flight. Because of apriori uncertainty of this component, it is appropriate to select the parameters of the shaping filter in such a way as to ensure the least impact on the accuracy of estimation. In other words, it is advisable in this case to receive a guaranteed result.

MM of the navigation field created by the GPS and GLONASS systems, including the visibility of individual satellites during the flight is also well characterized and can be implemented as it is described in the source [14, 19]. With the implementation of this model, as well as with the implementation of the receiver model, we shall further assume that we may use only code measurements: pseudo- range and pseudo-velocity. Next we shall assume a possibility to use dual-frequency measurements to practically exclude ionospheric and tropospheric delays, and the lack of selective access. With this approach, the main factor determining the possibility of GPS-navigation for the problem in question is the analysis of geometric visibility conditions of navigation satellites with the possible loss of communication, which is determined by the specific dynamics of the object. In this case, we shall assume that the uncertainty in searching a navigation constellation due to the Doppler shift of the carrier has already been overcome, and the receiver is synchronized in frequency, phase and code [1].

Now we shall move on to the analysis of the possible algorithms for processing navigation information. Due to the specific nature of the set task that requires processing of navigational measurements as soon as they are received, we will consider only the recursive modification of the following algorithms: Bayesian (and Kalman filter) or recurrent modification of the least square method, is not required as we know an additional apriori information about the state vector of the object. Thus, attention should be paid to the fact that an appropriate algorithm is to be implemented by the on board computer (OC) and, consequently, such operations as matrix inversion, summing of numbers with significantly different orders, etc should be excluded. Existing experience in this field $[20,21]$ suggests that the most appropriate modification of recursive algorithm for this task is one that will allow measurements as if bound to a definite time point by components. In this case, the result of processing the regular components of the measurement, "tied" to a given point in time, is seen as an apriori estimate in the processing of a subsequent component. Another important aspect in developing the processing algorithm is different speed with which navigation measurements enter. Thus, measurements generated in GINS enter with a relatively high frequency $(200 \mathrm{~Hz})$ while the code measurements from the receiver generally enter with a frequency of $1 \mathrm{~Hz}$ and the fact that GPS delays measurements may require special modifications of the recursive information algorithm. Finally, essential is the choice of a model predicting object's motion in the onboard algorithm. Moreover, generally there can be several different prediction models which will be used for different phases of flight: atmospheric and exo-atmospheric.

Next, the different prediction models can be used when using loosely coupled scheme of interconnection with the different rates of data entry from the GINS and GPS receiver.

Finally, the last aspect that we need to consider in setting the technical problem in the present paper is the selection of an approach to the shaping of an integrated navigation system for a space launch vehicle with GPS technology. It is important to stress once again, as mentioned earlier that the term "shape" will encompass the structure, composition, models and algorithms for integrated navigation system [1].

Obviously that with regard to the variety of different physical nature of uncontrolled factors having an effect 
within the framework of this problem, the nonlinear nature of $\mathrm{MM}$ of subject's motion and nonlinear relationship between the results of measurements and navigation components of the state vector, the only reasonable approach for solving the technical problems stated above is the simulation of the operation of the system to be shaped.

The above statement makes it necessary to create a special "tool" that shall ensure the implementation of the chosen approach to the solution of the technical problem set. This tool is a computer system with a fairly simple interface allowing, nevertheless varying interactively source data and parameters of the models and algorithms for analyzing and modeling results presented in graphic and numeric forms. Generally such a system must include two models: a model of the environment and a model of a launcher board.

In more detail, this problem will be discussed later in the chapter on modeling. Here we merely note that the model of a launcher board should include in addition to the model made by the navigation system, a model of the control channel, including steering signal formation and an actuating mechanism with the necessary detail level allowing the exploration of the impact of errors on the accuracy of the navigation controls.

For its part, a model of the environment should include as much detailed model of the object, disturbances, and natural and artificial navigation fields.

\section{A MODEL OF GUIDED MOTION OF A LAUNCH VEHICLE DURING THE POWERED PORTION OF FLIGHT}

This section presents developed models, algorithms and methods underlying the mathematical software designed for simulation of guided flight during the powered portion of the mission performed by a launch vehicle equipped with various navigation systems. The need to introduce the following material is determined, on the one hand, by peculiarities of the main power and disturbing factors of reducible models (operation of control loop by angular motion, calculation of the mass and inertial characteristics, modeling of thrust distribution), and, on the other hand, the detailed elaboration of a model to provide solution to the following tasks:

1. Simulation of the motion of the launch vehicle as a rigid body in space with 6 degrees of freedom, taking into account all the external disturbances.

2. The implementation of the control loop of the launch vehicle with engines having fixed and rotary nozzles which together with the drives are treated as dynamic systems.

3. Simulation of the work of measuring equipment and the on-board computer with regard to specific character of their operation as digital devices.

4. Operation of programs and algorithms developed to solve the target task by the launch vehicle (terminal control algorithms, navigation algorithm) in the framework of a model of the on-board computer.
5. Simulation of operation of the on-board integrated navigation system, taking into account a wide range of disturbing factors and errors made by measuring equipment.

In accordance with the above information for the sake of completeness further we shall give a brief, systematic description of coordinate systems, mathematical models of the launch vehicle's motion and disturbing factors, as well as of the algorithms used for complex simulation of the guided flight of the launch vehicle, will describe in detail peculiarities of methods and numerical procedures suitable for the specified objectives set before the development of mathematical software.

Structurally, the chapter consists of descriptions of movement patterns (unperturbed and perturbed motion) and loop control system that ensures fulfillment of program control on the basis of measurement and navigation data.

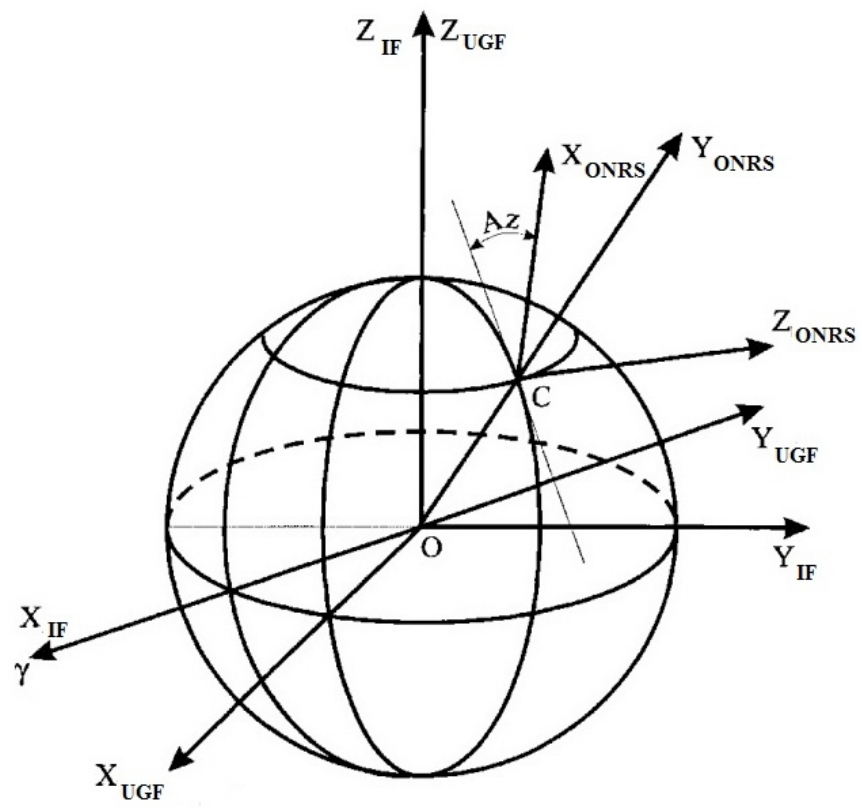

Fig. (6). Coordinate systems.

\subsection{Coordinate Systems}

To describe the motion of a launch vehicle, a control loop and GINS, we shall use the following coordinate system (Fig. 6):

\subsubsection{Absolute Inertial Frame (IF2000)}

Origin of IF2000 is in the center of mass of the Earth. Basic plane - mean equator is at $00^{\mathrm{h}} 00^{\mathrm{m}} 00^{\mathrm{s}}$ as on the 1 st of January 2010 (epoch J2010.0). Axis $X_{I F}$ is directed towards the average of the vernal equinox. Axis $Z_{I F}$ is directed along the axis of rotation of the Earth, relevant to the Conventional International Origin (CIO) 1900-1905. Axis $Y_{I F}$ complements the coordinate system to the right [14].

\subsubsection{Earth-Fixed Coordinate System (UGF)}

Origin of UGF is in the center of mass of the Earth. Axis $Z_{U G F}$ is directed along the axis of rotation of the Earth, relevant to the CIO 1900-1905. Axis $X_{U G F}$ passes through 
the Greenwich meridian, relevant to the CIO. Axis $Y_{U G F}$ complements the coordinate system to the right [21].

\subsubsection{Inertial Navigation Reference System (INRS)}

Origin of INRS is in the point of launching a launch vehicle. Axis $Y$ is directed along local vertical line. Axis $X$ makes an angle $A_{Z}$ with the direction to the north (azimuth of launch angle). Axis $Z$ complements the coordinate system to the right [22].

\subsubsection{Onboard Navigation Reference System (ONRS)}

This reference system (RS) must coincide with INRS at the moment of launching the launch vehicle and has to be kept to the selected axis directions during its flight. Sensitivity axes of accelerometers that measure the apparent velocity increment of the launch vehicle (basic information for INS) lie in the direction of the axes of the simulated RS [21].

\subsubsection{Body Frame $(B F)$}

Origin $\mathrm{BF}$ for a target flying vehicle is in its center of mass. Axes BF $\left(X_{C}, Y_{C}, Z_{C}\right)$ are axes of symmetry of the flying vehicle [21].

We shall present equations describing transitions between the Coordinate Systems used. With this purpose, we introduce operator matrices of rotation around each axis at an angle $\alpha$ :

$$
\begin{aligned}
& R_{X}(\alpha)=\left(\begin{array}{ccc}
1 & 0 & 0 \\
0 & \cos (\alpha) & \sin (\alpha) \\
0 & -\sin (\alpha) & \cos (\alpha)
\end{array}\right), \\
& R_{Y}(\alpha)=\left(\begin{array}{ccc}
\cos (\alpha) & 0 & -\sin (\alpha) \\
0 & 1 & 0 \\
\sin (\alpha) & 0 & \cos (\alpha)
\end{array}\right), \\
& R_{Z}(\alpha)=\left(\begin{array}{ccc}
\cos (\alpha) & \sin (\alpha) & 0 \\
-\sin (\alpha) & \cos (\alpha) & 0 \\
0 & 0 & 1
\end{array}\right) .
\end{aligned}
$$

Then the matrix of transition from the inertial to the Greenwich Coordinate System $A_{I F}^{U G F}$ is written as follows [3]:

$A_{I F}^{U G F}=R_{Z}(G S T)$,

where GST is Greenwich Sidereal Time.

Coordinates of the navigation satellites used by the system GPS, as well as navigational estimates of the launch vehicle's position and velocity supplied by the multi-channel GPS receiver are set in one of the World Geodetic Systems: WGS-84 or PP-90. To move from Greenwich Coordinate System to the Coordinate System, the following equation is used:

$$
X_{W G S-84}=A_{U G F}^{W G S-84} X_{U G F},
$$

where the matrix of transition from the Greenwich Coordinate System to the Coordinate System WGS-84 $A_{U G F}^{W G S-84}$ is written as follows [23]:

$A_{U G F}^{W G S-84}=\left(\begin{array}{ccc}1 & 0 & x_{p} \\ 0 & 1 & -y_{p} \\ -x_{p} & y_{p} & 1\end{array}\right)$,

$x_{p}, y_{p}$ are the current coordinates of a poleте.

Transition from INRS to WGS-84 is described by the following equation:

$$
\begin{aligned}
& X_{W G S-84}^{*}=A \times X_{U H C K}+X_{W G S-84}^{L P}, \\
& A^{T}=R_{Y}(-(90+A z)) R_{X}(\phi) R_{Z}(\lambda-90), \\
& X_{W G S-84}=A_{W G S-84}^{W G S-84} \cdot X_{W G S-84}^{*}, \\
& { }_{W G S-84}^{*}=R_{Z}\left(\Omega_{E a r t h} \cdot t\right),
\end{aligned}
$$

where $X_{W G S-84}^{*}$ is a launch vehicle's state vector in "the frozen" at the moment of start Coordinate System WGS-84; $X_{W G S-84}^{L P}$ is the position of the launch point in WGS-84.

The matrix of transition from INRS to the Body Frame $A_{\text {инск }}^{B F}$ is written as follows [3]:

$A_{\text {инСK }}^{B F}=A_{X}(\gamma) \times R_{Z}(\vartheta) \times R_{Y}(\psi)$,

where $\vartheta, \psi, \gamma$ are Euler angles of launch vehicle's orientation.

\subsection{The Model of the Unperturbed Motion}

In order to give a systematic presentation below we shall give a full MM of unperturbed motion of the center of mass of the launch vehicle and angular motion the launch vehicle in an active phase. When considering the unperturbed motion, we take into account the following power factors:

1. the attracting force of the Earth, taking into account the non-spherical potential up to the 4th degree and order including;

2. thrust of the launch vehicle according to the thrust nominal profile and fuel mass flow;

3. aerodynamic force in accordance with the parameters of the dynamic environment and coefficients of drag force and lift specified in a table.

Furthermore when considering the unperturbed motion of a launch vehicle we assume that the assembly of the launch vehicle has been carried out without errors. All angular and linear parameters correspond to the nominal levels and there are no disturbing moments during separation of the stages.

\subsection{The Equations of Motion of the Center of Mass}

We shall use the MM of the launch vehicle's center mass motion based on the laws of Newtonian mechanics, according to which the model of the motion of a point particle in INRS is written as follows [3]: 
$m \ddot{X}=\sum_{i} F_{i}$,

where $m$ is the mass of the launcher;

$X$ is the launcher position of the vector;

$F_{i}$ are the forces acting on the launcher.

In the above models the accuracy of the motion of the center of mass of the launch vehicle is determined by a composition of forces to be taken into account during the simulation on the basis of the duration of the active phase and the need of the simulation accuracy. As it was noted above, when considering the unperturbed motion we take into account the following power factors:

- the attracting force of the Earth, taking into account the non-spherical potential up to the 4th order including;

- $\quad$ thrust of the propulsion system of the launch vehicle according to the thrust nominal profile and fuel mass flow;

- $\quad$ aerodynamic force in accordance with the parameters of the dynamic environment and coefficients of drag force and lift specified in a table.

Let us consider each of these factors separately.

\subsubsection{Gravity}

For representation let's expand the geo-potential into spherical functions [24]:

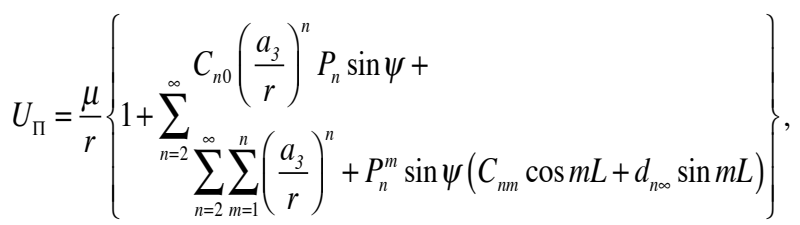

where $\mu=f M$ is a product of gravitation constant by terrestrial mass;

$r, L, \psi$ are respectively the geocentric radius, longitude and latitude of the considered point in WGS-84;

$P_{n} \sin \psi$ is Legendre polynomial of order $\mathrm{n}$;

$P_{n}^{m} \sin \psi$ are associated spherical functions;

$C_{n 0}, C_{n m}, d_{n m}$ are dimensionless constants which characterize the form and the gravitational field of the Earth.

Radial, meridional and normal components of the acceleration of gravity can be calculated according to the following formulae:

$g_{r}=\frac{\partial U_{T}}{\partial r}, g_{\psi}=\frac{1}{r} \frac{\partial U_{T}}{\partial \psi}, g_{L}=\frac{1}{r \cos \psi} \frac{\partial U_{T}}{\partial L}$.

It should be noted that the integration of the equations of motion of the center of mass in the launch vehicle is done in INRS, while the calculation of the acceleration conditioned by the gravity of the Earth is conducted in WGS-84 in spherical coordinates. Accordingly, in order to determine the projection of the acceleration on the axis of INRS we must [25]:

1. Determine the coordinates of the center of mass of the launcher in UGF in accordance with equations (3).

2. Calculate the spherical coordinates of the center of mass of the launch vehicle in UGF:

$$
\begin{aligned}
& r=\sqrt{x^{2}+y^{2}+z^{2}}, \\
& L=\operatorname{arctg} \frac{y}{x}, \\
& \psi=\operatorname{arctg} \frac{z}{\sqrt{x^{2}+y^{2}}} .
\end{aligned}
$$

1. Determine projection of the acceleration of the spherical WGS-84, using the following equation:

$$
\left(\begin{array}{c}
x=r \cdot \cos L \cdot \cos \psi \\
y=r \cdot \sin L \cdot \cos \psi \\
z=r \cdot \sin \psi
\end{array}\right)
$$

2. Get the projections of acceleration in INRS, in accordance with equations (3).

\subsubsection{Thrust}

Rocket thrust is generated on account of combustion of fuel with mass flow rate $m_{s}$ and discharge of combustion products through the nozzles at flow rate $W$.

Thrust at a certain altitude $h$ is determined by the following subjection [7]:

$P(h)=m_{s} W+S_{a}\left(p_{a}-p_{h}\right)$,

where $p_{a}$ is pressure at the nozzle exit; $p_{h}$ is pressure at a given height h; $S_{a}$ is nozzle exit area.

At sea level where $p_{h}=p_{o}$, thrust is minimal:

$P(0)=P_{0}=m_{s} W+S_{a}\left(p_{a}-p_{h}\right)$.

In vacuum where $p_{h}=0$, thrust reaches its maximum:

$P_{V}=m_{s} W+S_{a} p_{a}$.

Then the expression for $P(h)$ shall be rewritten as follows:

$P(h)=P_{V}-S_{a} p_{h}$.

The difference between thrusts at sea level and in vacuum shall be $P_{V}-P_{0}=S_{a} p_{0}$ dependent on nozzle exit $S_{a}$, determining the engine critical altitude, i.e. its ability to most effectively work in a rarefied atmosphere. The value $S_{a}$ determines the expansion ratio of a jet of flowing through the nozzle gases and, consequently, the pressure $p_{a}$ in the nozzle exit.

An important characteristic of the efficiency of the engine is the specific thrust, i.e. a ratio of thrust to fuel consumption per second [14]: 
$P_{y \partial}(h)=\frac{P(h)}{m_{S} g_{0}}=P_{y \partial_{\Pi}}-\frac{p_{b} S_{a}}{m_{S} g_{0}}$,

where specific thrust in «vacuum»: $P_{y \partial_{\Pi}}=\frac{W}{g_{0}}+\frac{p_{0} S_{a}}{m_{S} g_{0}}$.

The so-called "earth" specific thrust is always less than the specific thrust in vacuum:

$P_{y \partial_{0}}=P_{y \partial_{\Pi}}-\frac{p_{0} S_{a}}{m_{S} g_{0}}$.

Attractive force direction is determined in Body Frame related to a coordinate system independent of the orientation of the longitudinal axis of the nozzle relative to the longitudinal axis of the engine of the launch vehicle. When considering the unperturbed motion of the launch vehicle, angles of incidence of the engine are considered nominal and the direction is determined only by the nozzle deflection angles resulting from tracking.

It should be noted that when calculating attractive force, nominal profiles of thrusts in vacuum and the nominal fuel mass flow rate specified in the table (Fig. 7) are used.

Since the integration of the equations of motion of the center of mass of the launch vehicle is made in INRS, while calculation of accelerations caused by thrust generated by the propulsion system is carried out in BF, it is necessary to convert projections of forces from BF into INRS according to a transition matrix in order to determine projections of the acceleration on INRS axis (4).

\subsubsection{Aerodynamic Forces}

Aerodynamic forces are the result of the impact of the environment on the surface of the launch vehicle when it moves. They are defined as the sum of the elementary tangential and normal forces acting on the body of the launch vehicle. Depending on whether the moving body is symmetrical relative to the axis, or its axis of symmetry is directed in the motion along the velocity vector or deviates from it, there appears one axial force $X_{B F}$ or one normal force $Y_{B F}$. Generally in BF [22]:

$X_{B F}=c_{x_{i}} q S$,

$Y_{B F}=c_{y_{i}} q S=c_{y_{i}}^{\alpha} q S \alpha$,

where $c_{x_{i}}, c_{y_{i}}, c_{y_{i}}^{\alpha}$ are dimensionless aerodynamic coefficients;

$q=\frac{\rho V^{2}}{2}$ is dynamic pressure;

$\rho$ is air density at a given point of the trajectory;

$S$ is a midsection area.

More common is the idea of the aerodynamic forces in the form of frontal drag $X$ and lift $Y$, directed accordingly along stream velocity and perpendicular thereto:

$$
\begin{aligned}
& X=c_{x} q S, \\
& Y=c_{y} q S=c_{y}^{\alpha} q S \alpha,
\end{aligned}
$$

where $c_{x}, c_{y}, c_{y}^{\alpha}$ are coefficients similar to $c_{x_{i}}, c_{y_{i}}, c_{y_{i}}^{\alpha}$, but referred to a coordinate system whose axes are oriented along the stream perpendicular thereto.

It is obvious that there are simple dependences between $X_{B F}, Y_{B F}$ and $X, Y$ :

$X=X_{B F} \cos \alpha+Y_{B F} \sin \alpha$,

$Y=-X_{B F} \sin \alpha+Y_{B F} \cos \alpha$,

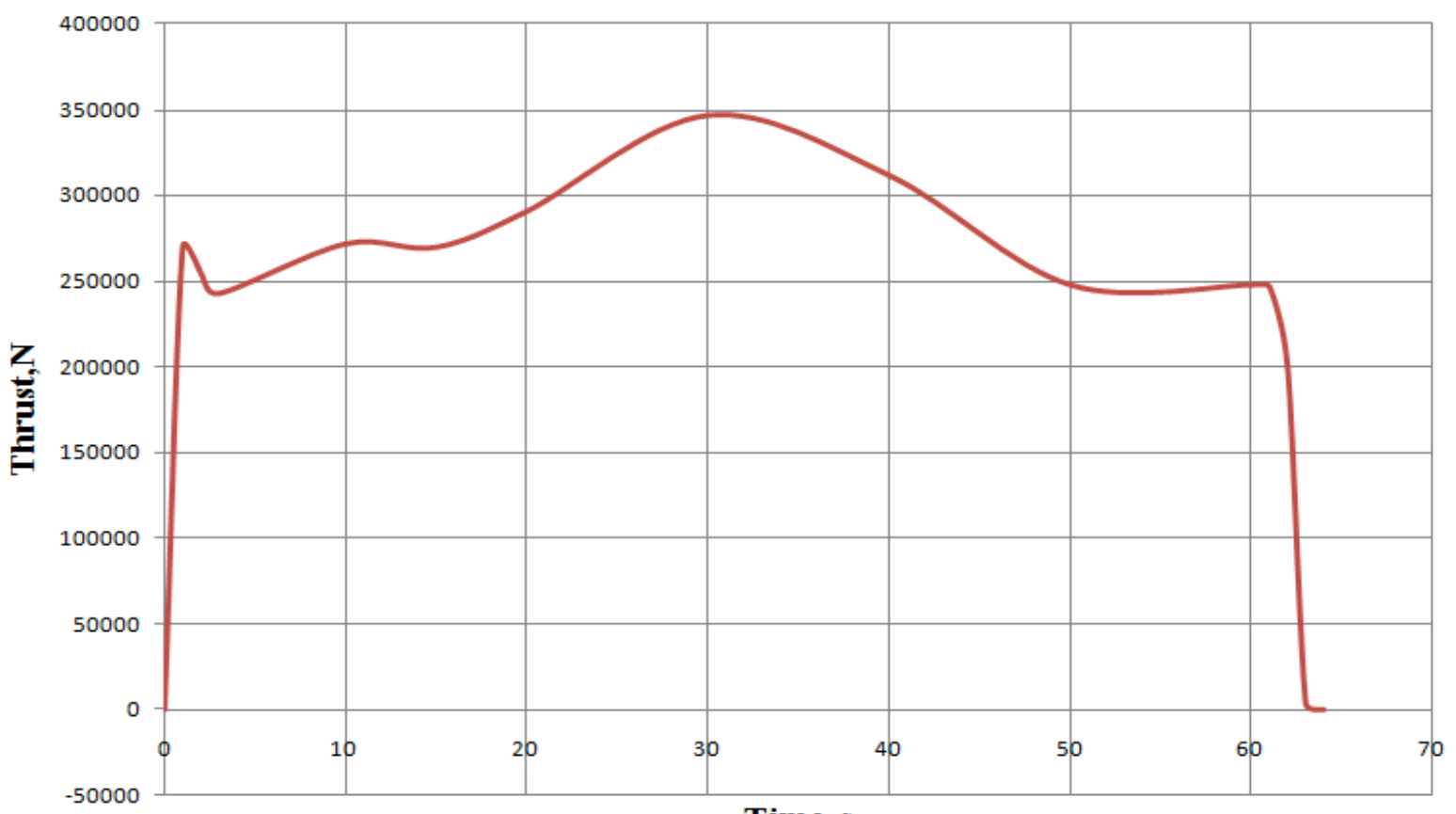

Time, $\mathrm{s}$

Fig. (7). Thrust profile for the propulsion system of a launch vehicle. 
or

$X_{B F}=X \cos \alpha-Y \sin \alpha$,

$Y_{B F}=X \sin \alpha+Y \cos \alpha$.

These formulas are valid for small angles of attack, which are present during the flight of the launch vehicle in the dense layers of the atmosphere. Therefore, if we suggest $\sin \alpha=\alpha, \cos \alpha=1$, the dependence can be simplified:

$X=X_{B F}+Y_{B F} \alpha=-X_{B F} \alpha+Y_{B F}$,

$X_{B F}=X-Y, Y_{B F}=X \alpha+Y$.

It must be noted that when calculating the aerodynamic forces we use nominal coefficients of drag and lift specified in a table.

Furthermore, since the projections of the aerodynamic forces are calculated in a coordinate system connected with the air speed of the launch vehicle, it is necessary to determine a single vector of air speed of the launch vehicle $V_{B}^{0}$ in INRS. Since the launch vehicle performs its mission in the dense layers of the atmosphere which rotates with the Earth, the air velocity vector in INRS is defined as follows [22]:

$V_{B}^{0}=V+A_{U G F 84}^{I N R S} \cdot\left[\Omega_{3} \times R\right]$,

where $V$ is the launcher's velocity vector in INRS; $\Omega_{3}-$ angular velocity vector of rotation of the Earth in WGS 84; $R-$ radius vector of the launch vehicle; $A_{U G F 84}^{I N R S}$ is a matrix for conversion from WGS 84 into INRS.

To describe the unperturbed motion of the center of mass of the launch vehicle besides equations (5), it is necessary to use an equation of launcher mass change during the flight $m=M(t)$.

However, due to table dependence of the propulsion system's fuel mass flow per second and spasmodic changes in the mass during the separation of stages of the launch vehicle, the launch vehicle's full weight is calculated as the sum of all current stages with fuel in operating engines, determined in accordance with the current propulsion system's profile of thrust.

\subsection{The Equations of Rotational Motion of the Launch Vehicle}

The spatial angular motion of the center of mass of the launcher is described by the following equations projected on the axes BF $[14,26]$ :

$$
\begin{aligned}
& \omega_{x}=\frac{\sum M_{x}}{I_{x}} ; \\
& \omega_{Y}=\frac{\left(I_{Z}-I_{X}\right) \omega_{x} \omega_{Z}}{I_{Z}}+\frac{\sum M_{Y}}{I_{Y}} ; \\
& \omega_{Z}=\frac{-\left(I_{Z}-I_{X}\right) \omega_{x} \omega_{Y}}{I_{Z}}+\frac{\sum M_{Z}}{I_{Z}} .
\end{aligned}
$$

In these equations: $\omega_{x}, \omega_{Y}, \omega_{Z}$ are components of the angular velocity vector of the launch vehicle $\omega$ relative to axes
$\mathrm{BF} ; \sum M_{x}, \sum M_{Y}, \sum M_{Z}-$ components of the sum vector of moments $\sum M$, acting on the launch vehicle during the mission projected on axes BF; $I_{x}, I_{Y}, I_{Z}$ are axial moments of inertia of the launch vehicle (the launch vehicle's centrifugal moments of inertia are equal to zero because of symmetry of the object).

It should be noted that in order to connect angular motion parameters, i.e. a component of angular velocity vector $\omega$ in BF with the parameters of the center of mass of the launch vehicle (i.e. determining the orientation angles of the launch vehicle) we shall use an approach based on the Rodrigues-Hamilton parameters instead of the standard kinematic equations [7]

$$
\begin{gathered}
\dot{\gamma}=\omega_{x}+\operatorname{tg} \psi\left(\omega_{y} \sin \gamma+\omega_{z} \cos \gamma\right), \\
\vartheta=\left(\omega_{y} \sin \gamma+\omega_{z} \sin \gamma\right) \frac{1}{\cos \psi} \\
\psi=\omega_{y} \cos \gamma-\omega_{z} \sin \gamma
\end{gathered}
$$

This approach is based on the idea of finite rotation of a rigid body within its own quaternion of conversion between coordinate systems whose components are called the Rodrigues-Hamilton parameters.

In line with this approach, the Rodrigues-Hamilton parameters are connected with the Euler angles via the following relations $[20]$ :

$$
Q=\left(\begin{array}{c}
\cos \left(\frac{\vartheta}{2}\right) \\
0 \\
0 \\
\sin \left(\frac{\vartheta}{2}\right)
\end{array}\right) \circ\left(\begin{array}{c}
\cos \left(\frac{\psi}{2}\right) \\
0 \\
\sin \left(\frac{\psi}{2}\right) \\
0
\end{array}\right) \circ\left(\begin{array}{c}
\cos \left(\frac{\gamma}{2}\right) \\
\sin \left(\frac{\gamma}{2}\right) \\
0 \\
0
\end{array}\right),
$$

where $Q$ is own quaternion of conversion between INRS and $\mathrm{BF}$; ० is quaternion multiplication sign.

Transition matrix $A_{I N R S}^{B F}$ between INRS and BF is made on the basis of its own quaternion of conversion in accordance with the following formulae:

$$
A_{I F}^{B F}=\left(\begin{array}{ccc}
\frac{q_{0}^{2}+q_{1}^{2}-q_{2}^{2}-q_{3}^{2}}{|Q|} & \frac{2\left(q_{1} \cdot q_{2}+q_{0} \cdot q_{3}\right)}{|Q|} & \frac{2\left(q_{1} \cdot q_{3}-q_{0} \cdot q_{2}\right)}{|Q|} \\
\frac{2\left(q_{1} \cdot q_{2}-q_{0} \cdot q_{3}\right)}{|Q|} & \frac{q_{0}^{2}+q_{2}^{2}-q_{1}^{2}-q_{3}^{2}}{|Q|} & \frac{2\left(q_{2} \cdot q_{3}+q_{0} \cdot q_{1}\right)}{|Q|} \\
\frac{2\left(q_{1} \cdot q_{3}+q_{0} \cdot q_{2}\right)}{|Q|} & \frac{2\left(q_{2} \cdot q_{3}-q_{0} \cdot q_{1}\right)}{|Q|} & \frac{q_{0}^{2}+q_{3}^{2}-q_{1}^{2}-q_{2}^{2}}{|Q|}
\end{array}\right),
$$

where $q_{1}, q_{2}, q_{3}, q_{4},|Q|$ are components and a module of its own quaternion of conversion $Q$.

Traditional Euler angles can be defined on the basis of the present transition matrix [20] as follows 


$$
\left.\begin{array}{c}
\vartheta=\operatorname{arctg}\left(\frac{a_{12}}{a_{11}}\right), \\
\psi=\operatorname{arctg}\left(\frac{-a_{13}}{\sqrt{a_{11}^{2}+a_{12}^{2}}}\right), \\
\gamma=\operatorname{arctg}\left(\frac{a_{23}}{a_{33}}\right)
\end{array}\right\},
$$

where $a_{i j}$ is the component of the matrix $A_{I F}^{B F}$.

Then the kinematic equations, i.e. equations relating to the angular velocity vector of a rigid body with time derivatives of the kinematic parameters shall be written as follows [20]:

$Q=\frac{1}{2} \omega \circ Q$

This approach, compared with classical kinematic equations (14), due to linearity of equations (18) allows obtaining a high-precision stable numerical solution devoid of singular points.

Thus, the complete system of differential equations that describe the spatial motion of a flying vehicle, consists of 6 equations of the center of mass motion (5), 3 equations of an aircraft's angular motion (13) and 4 kinematic equations (18) describing the dynamics of the Rodrigues-Hamilton parameters.

Let us consider the method of generation of the total moment relative to the center of gravity acting on the launch vehicle. Obviously, the total moment is the vector sum of the moments created by active and passive forces acting on the launch vehicle. Let's begin with gravity.

Designers of a launch vehicle try to position the center of mass at its geometrical axis in such a way that thrust does not create disturbing moment about the center of mass. We shall assume that the center of mass lies exactly on the longitudinal axis of the launch vehicle at a distance $x_{L M}$ from the top. However, since fuel mass flow per second of the propulsion system depends on values specified in a table as well as on abrupt changes in the mass and length during the separation stages with the current engine, operating fuel mass is determined in accordance with the current thrust profile and stage joint angles [21].

Since gravity always acts along a straight line passing through the center of mass, it does not create a moment.

\subsubsection{Aerodynamic Moments}

Since force along its line of action can be moved to any point, we shall agree to assume aerodynamic force applied in the center of pressure of the launch vehicle. In this case, the force components acting along the axes $X_{B F}, Y_{B F}$ of BF or along the axes $X, Y$ of velocity system can also be considered to be applied in the center of pressure. Force $X_{B F}$ acts along the longitudinal axis of the launch vehicle and does not create a moment about the center of mass. Force $Y_{B F}$ creates a moment about the center of mass [22]:

$M_{Z}^{A D}=Y_{B F}\left(x_{\text {цд }} \cdot x_{\text {ЦМ }}\right)=c_{y 1}^{\alpha} q S\left(x_{\text {ЦД }} \cdot x_{\text {ЦM }}\right) \alpha$.

It must be noted as well that the position of the center of pressure is specified in a table in accordance with the current flight time and abruptly changes during separation of stages of the launch vehicle.

\subsubsection{Thrust Moment of the Engine}

Control moments must provide the ability to control the longitudinal motion of the vehicle and angular movements about axes of $\mathrm{BF}(0 x-$ rolling, $0 y-$ yaw, $0 z-$ pitching motion).

The launch vehicle under consideration has no velocity control due to the peculiarities of the propulsion system and control algorithms, i.e. amount of engine thrust is realized "in fact" without affecting the control system. In this case, the main means of control is to change the direction of the thrust vector to generate thrust moment about the center of mass of the launch vehicle in one of the following channels [21]:

- roll channel is a channel to control angular motion about body axis $0 x$;

- yaw channel is a channel to control angular motion about the body axis $0 y$;

- $\quad$ itch channel is a channel to control angular motion about the axis $0 z$.

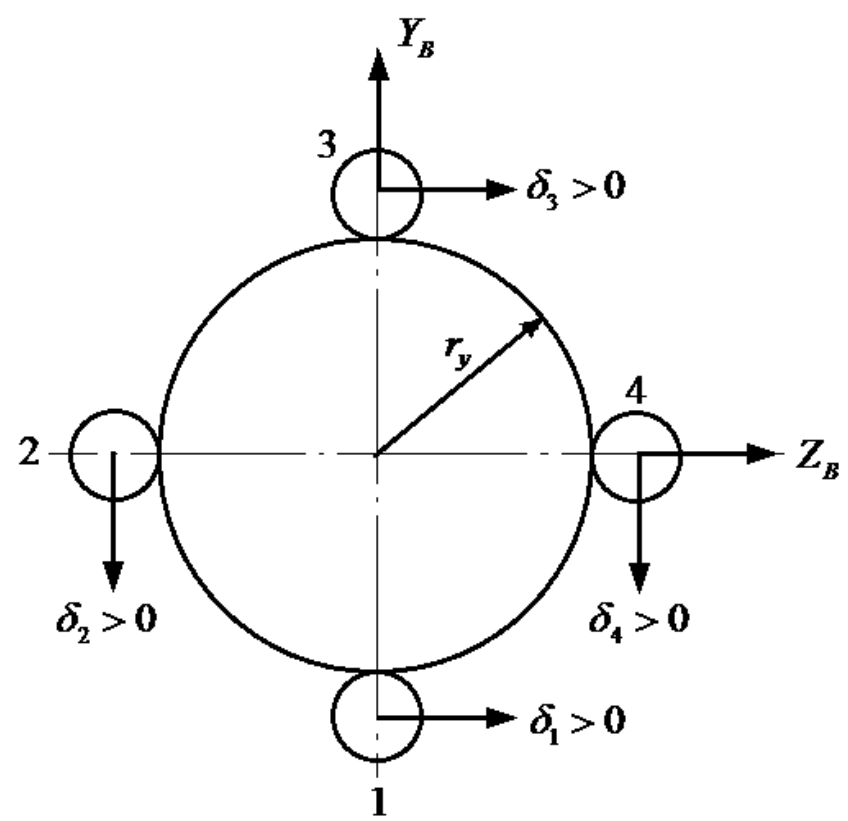

Fig. (8). Scheme for creating control moments of the launch vehicle.

Let's consider a typical way to control roll with four rotary propulsion control motors with one rotation axis (the 
so-called stage I boosters). The positive direction of rotation about the roll axis is determined by the mark of the control moment. It creates a positive rolling control moment:

$M_{x}=\operatorname{Pr}_{y}\left(\cos \delta_{1}+\cos \delta_{2}-\sin \delta_{3}-\sin \delta_{4}\right)$,

where $P$ is the thrust of one engine; $r_{y}$ is an arm of engines thrust about the center of mass.

To create yaw control moments, engines situated in planes 1-3 (Fig. 8) synchronously deviate at angles $\delta_{1}, \delta_{3}$ :

$M_{y}=-P l_{y}\left(\sin \delta_{1}+\sin \delta_{3}\right)$,

where $l_{y}$ is a distance from the center of mass to the axis of engine rotation.

To create pitch control moments, engines situated in planes 2-4 (Fig. 8) synchronously deviate at angles $\delta_{2}, \delta_{4}$ :

$$
M_{z}=-P l_{y}\left(\sin \delta_{2}+\sin \delta_{4}\right) \text {. }
$$

It should be noted that the calculation of arms of force, i.e. a distance from the center of mass to the axis of rotation of the engine, the current position of the center of mass and the arm is determined based on the current angles of stages and the engine setting.

\section{CONCLUSIONS}

1. Based on the above, we have set a technical problem of the conceptual design of an integrated navigation system for the space launch vehicle qualified to inject small artificial Earth satellites into low and medium circular orbits.

2. The conceptual design of the integrated navigation system based on GPS technology involves determination of its structure, models and algorithms, providing the required accuracy and reliability in injecting payloads with due regard to restrictions on weight and dimensions of the system.

3. We have defined the sequence of essential scientific and technical problems that lead to the solution of the major technical problem. This sequence includes:

a) selection of quality (accuracy) criteria for solving the navigation task;

b) selection of a method for integration of navigation information;

c) making a model of an object's motion, GINS, navigation field, GPS receiver, taking into account all uncontrolled factors;

d) making a "tool" to simulate functioning of the system involved.

4. It has been demonstrated that it is appropriate to take a posteriori accuracy dispersion of the position and velocity vectors of the launch vehicle in phases of flight of I-III stages as a criterion of accuracy of solving a navigation task.

5. We have made an analysis of possible models of flight and navigation measurements and identified key potential difficulties in the process of their creation.

\section{CONFLICT OF INTEREST}

The author confirms that this article content has no conflict of interest.

\section{ACKNOWLEDGEMENTS}

Declared none.

\section{REFERENCES}

[1] Z. Nickolay, "Integrated navigation system for prompting of the commersial carrier rocket", In: Information Technology Conference for Academia and Professionals (ITC-AP 2013), Sharda University, India, 19-21 April, 2013.

[2] Flight instruments and navigation systems/ Politecnico di Milano Dipartimento di Ingegneria Aerospaziale, Aircraft systems (lecture notes), version, 2004.

[3] G. M. Siouris, Aerospace Avionics Systems. Academic Press: INC 1993.

[4] K-S. Choi, "Development of the commercial launcher integrated navigation system the mathematical model, using GPS/GLONASS technique", In: 52nd International Astronautical Congress, France, Toulouse, 2001.

[5] Available at: http://ru.wikipedia.org/wiki/ - Vega launcher (Вега ракета-носитель), (in Russian).

[6] G. Siharulidze Yu, Flying vehicles ballistics, Nauka Publisher, Russia, 1982, p. 351, (Сихарулидзе Ю.Г. Баллистика летательных аппаратов. М.:Наука, 351с., 1982).

[7] A. A. Lebedyev, G. G. Adzhimamudov, V. N. Baranov, and V. T. Bobronnikov, Fundamentals of flying vehicles systems synthesis, Moscow Aviation Institute MAI: Moscow, 1996, p. 224, (Лебедев А.А., Аджимамудов Г.Г., Баранов В.Н., Бобронников В.Т. и др. Основы синтеза систем летательных аппаратов. М.: МАИ, 224 c., 1996).

[8] D. J. Bayley, Design optimization of space launch vehicles using a genetic algorithm, Auburn University, Alabama, 2007, p. 196.

[9] B. Scherzinger, "Precise Robust Positioning with Inertial/GPS RTK", In: Proceedings of ION-GPS-2000, Salt Lake City UH September 20-23, 2000.

[10] C. Urmson, C. Ragusa, and D. Ray, "A robust approaches to high speed navigation for unrehearsed Desert Terrain", J. Field. Rebot. vol. 23, no. 8, August, 2006, pp. 467-508, 2006

[11] W. Whittaker and L. Nastro, "Utilization of position and orientation data for preplanning and real time autonomous vehicle navigation", GPS World, Sept 1, 2006.

[12] J. Daniel, Biezad integrated navigation and guidance systems, AIAA Education Series, American Institute of Aeronautics and Astronautics : USA, 1999.

[13] J. F. Hanaway and R. W. Moorehead, Space shuttle avionics system, National Aeonatics ans Space Administration Office of Management, Scientific and Technical Information Division: USA, p. 505,1989 .

[14] M. N. Krasilshikov, and G. G. Serebryakov, Control and guidance of unmanned flying vehicles on the basis of modern information technologies, Fizmatlit Publisher: Moscow, 2003, p. 280. (Управление и наведение беспилотных маневренных летательных аппаратов на основе современных информационных технологий / Под ред. М.Н. Красильщикова и Г.Г. Себрякова. - М.: ФИЗМАТЛИТ, 280 с., 2003).

[15] A.J. Kelly, "A $3 D$ state space formulation of a navigation Kalman filter for autonomous vehicles", Report CMU-RI-TR-94-19, CMU Robotics Institute Technical, PA, USA, 1994

[16] A. Kelly, "Modern inertial and satellite navigation systems". Technical Rep, CMU-RI-TR-94-15, The Robotics Institute Carnegie Mellon University, USA, 1994.

[17] M. Horemu, Integrated Navigation. Royal Institute of Technology, Stocholm, 2006 
[18] D. B. Cox Jr., Integration of GPS with inertial navigation systems, Institute of Navigation, USA, pp. 144-153, 1978.

[19] Available at: http://satellite-monitoring.atcommunication. com/en/s ecure/seu8800.pdf - Satellite Monitoring and Intercept System SEU 8800.

[20] E. A. Fedosov, V. T. Bobrronnikov, M. N. Krasilshikov, and V. I. Kuhtenko, Dynamical design of automatic control systems of flying vehicles, Mashinostroyeniye Publisher: Moscow, 1977, p. 336, (Федосов Е.А., Бобронниковв.Т., Красильщиков М.Н., Кухтенко В.И. и др. Динамическое проектирование систем управления автоматических маневренных летательных аппаратов. М.: Машиностроение, 336 с., 1977).

[21] V. V. Malyshev, M. N. Krasilshikov, V. T. Bobronnikov, and V. D. Dishel, Aerospace vehicle control, 1996.

[22] T. R. Kane, P. W. Likins, and D. A. Levinson, Spacecraft Dynamics, The Internet-First University Press: Cornell University, p. $454,2005$.
[23] C. L. Botasso, "Solution procedures for maneuvering multibody dynamics problems for vehicle models of varying complexity", Multibody Dyn. Comput. Methods Appl. Sci., vol. 12, pp. 57-79, 2008.

[24] A. Prati, S. Calderara, and R. Cuccira, Using circular statistics for trajectory shape analysis, University of Modena and Reggio Emilia: Italy. Available at: http://mplab.ucsd.edu/wp-content/uploa ds/cvpr2008/ conference/data/papers/497.pdf

[25] B. F. Zhdanyuk, Fundamentals of statistical processing of trajectory measurements, Mashinostroyeniye Publisher: Moscow, p. 384, 1978 (Жданюк Б.Ф. Основы статистической обработки траекторных измерений. М.: Машиностроение, 384 с., 1978).

[26] H. S. Tsien, T. C. Adamson, and E. L Knuth, "Automatic rocket navigation of a long range rocket vehicles", J. Am. Soc., JulyAugust, 1952.

(C) Nickolay Zosimovych; Licensee Bentham Open.

This is an open access article licensed under the terms of the Creative Commons Attribution Non-Commercial License (http: //creativecommons.org/licenses/by$\mathrm{nc} / 3.0 /$ ) which permits unrestricted, non-commercial use, distribution and reproduction in any medium, provided the work is properly cited. 